\title{
ESSENTIAL OIL COMPOSITION OF THE FLOWERS OF Plumeria rubra cv. acutifolia FROM CHINA
}

\author{
Shuai Zhang, ${ }^{1}$ Ji Dong, ${ }^{1}$ and Hao Cheng ${ }^{2 *}$
}

The genus Plumeria (Apocynaceae) originated from Central America and consists of about eight species of which many are widely distributed in tropical countries [1]. Plumeria rubra L. cv. acutifolia, one of the two species of the genus occurring in China, is a small tree whose flowers are used to treat cold and fever, whooping cough, tracheitis, infective hepatitis, diarrhea, calculus of urethra, and mastitis [2].

In this report, the essential oil was obtained from the flowers of Plumeria rubra cv. acutifolia. collected from Zhaoqing City, Guangdong Province of China by supercritical $\mathrm{CO}_{2}$ extraction [3]. The chemical composition of the essential oil was subsequently analyzed by GC/MS.

Thirty-one compounds were identified, representing $88.42 \%$ of the total oil. The major compounds are ( \pm -trans-nerolidol (17.28\%), hexadecanoic acid (11.47\%), tetradecanoic acid (10.68\%), $\beta$-linalool (8.86\%), octadecenoic acid (5.87\%), and cis-linoleic acid (4.68\%) (Table 1). Further investigation of the flowers of Plumeria rubra L. cv. acutifolia essential oil is still needed.

TABLE 1. Essential Oil Composition of the Flowers of Plumeria rubra. cv. Acutifolia

\begin{tabular}{|c|c|c|c|c|c|}
\hline Compound & $\mathrm{Rt}, \min$ & $\%$ & Compound & $\mathrm{Rt}, \mathrm{min}$ & $\%$ \\
\hline Benzene acetaldehyde & 6.563 & 0.24 & Benzyl benzoate & 28.271 & 1.35 \\
\hline cis-Linalool oxide & 7.468 & 0.73 & Tetradecanoic acid & 28.605 & 10.68 \\
\hline trans-Linalool oxide & 8.102 & 0.27 & Tetradeeanoic acid, ethyl ester & 29.123 & 1.05 \\
\hline$\beta$-Linalool & 8.365 & 8.86 & Hexadecanol & 29.607 & 0.95 \\
\hline$\beta$-Citral & 9.541 & 1.14 & Nonadecane & 30.984 & 0.26 \\
\hline cis-Geraniol & 11.256 & 0.85 & Hexadecanoic acid & 31.506 & 11.47 \\
\hline trans-Geraniol & 12.958 & 3.48 & Hexadecanoic acid, ethyl ester & 31.823 & 4.12 \\
\hline Geranial & 14.178 & 0.62 & 3,7,11-Trimethyl-2,6,10- & 33.022 & 1.18 \\
\hline 2,4-Bis(1,1-dimethylethyl)-5-methyl-phenol & 17.369 & 0.18 & dodecatrien-1-ol & & \\
\hline$( \pm)$-trans-Nerolidol & 20.556 & 17.28 & Tetradecanol & 34.119 & 1.98 \\
\hline Dodecanoic acid & 21.443 & 0.69 & (S)-4,4-Dimethyl-2-(4-methyl-3- & 34.401 & 0.23 \\
\hline Squalene & 22.281 & 0.55 & cyclohexen-1-yl)-1,1,5-hexadiene & & \\
\hline (E)-Farnesene epoxide & 24.372 & 0.78 & Heneicosane & 35.638 & 0.57 \\
\hline$\alpha$-Bisabolol & 25.303 & 1.29 & cis-Linoleic acid & 36.578 & 4.68 \\
\hline 1,6,10-Dodecatrien-3-ol, 3,7,11-trimethyl- & 25.785 & 0.36 & Linolenic acid, methyl ester & 36.796 & 2.76 \\
\hline cis-Farnesal & 26.697 & 3.31 & Octadecenoic acid & 37.686 & 5.87 \\
\hline trans-Farnesal & 27.428 & 0.64 & Total & & 88.42 \\
\hline
\end{tabular}

\section{REFERENCES}

1. G. Ye, Y. L. Yang, G. X. Xia, M. S. Fan, and C. G. Huang, Magn. Reson. Chem., 46, 1195 (2008).

2. Chinese Materia Medica, Shanghai Science \& Technology Press, Shanghai, China, 1999.

3. $\quad$ F. J. Eller and J. W. King, Phytochem. Anal., 11, 226 (2000).

1) College of Chemistry and Chemical Engineering of Zhaoqing University, Zhaoqing, 526061, Guangdong, P. R. China; 2) Department of Biological and Chemical Engineering, Guangxi University of Technology, Liuzhou, 545006, Guangxi, P. R. China, fax: +86 772 2685375, e-mail: hc0229@live.com. Published in Khimiya Prirodnykh Soedinenii, No. 1, January-February, 2016, p. 133. Original article submitted February 28, 2014. 